\title{
Synthesis and Characterization of CuO Nanoparticles and CuO Doped PVA Nanocomposites
}

\section{Alabur Manjunath*, Mohammed Irfan, Kabbinadavamsha Prasannakumar Anushree, Kuruba Mahesha Vinutha, Narasimhappa Yamunarani}

Department of P.G. Studies in Physics, Government Science College, Chitradurga, India

Email: *manjugsc@yahoo.com

How to cite this paper: Manjunath, A., Irfan, M., Anushree, K.P., Vinutha, K.M. and Yamunarani, N. (2016) Synthesis and Characterization of $\mathrm{CuO}$ Nanoparticles and $\mathrm{CuO}$ Doped PVA Nanocomposites. Advances in Materials Physics and Chemistry, 6, 263-273.

http://dx.doi.org/10.4236/ampc.2016.610026

Received: September 2, 2016

Accepted: October 16, 2016

Published: October 19, 2016

Copyright $\odot 2016$ by authors and Scientific Research Publishing Inc. This work is licensed under the Creative Commons Attribution International License (CC BY 4.0).

http://creativecommons.org/licenses/by/4.0/

\section{(c) (i) Open Access}

\begin{abstract}
$\mathrm{CuO}$ nanoparticles were synthesized by aqueous precipitation method and $\mathrm{CuO}$ doped PVA nanocomposites ( $1 \mathrm{wt}, 2 \mathrm{wt}, 3 \mathrm{wt}, 4 \mathrm{wt}$ and $5 \mathrm{wt}$ ) were prepared by solution casting method. The pellets of $\mathrm{CuO}$ nanoparticles and films of $\mathrm{CuO}$ doped PVA nanocomposites were used for electrical studies in the frequency range of $50 \mathrm{~Hz}$ to 5 $\mathrm{MHz}$ and in the temperature range of 303 to $338 \mathrm{~K}$. The dielectric constant decreases while the AC electrical conductivity increases with increasing frequency and also with increase in temperature. XRD study confirms the crystalline nature of $\mathrm{CuO}$ nanoparticles and the average crystallite size is found to be around $8 \mathrm{~nm}$. The peak widths in XRD pattern of PVA-CuO nanocomposites are broadened by incremental addition of $\mathrm{CuO}$ nanomaterials which implies an increase in the amorphous character of PVA-CuO nanocomposites that result in greater ionic diffusivity and high ionic conductivity. It is clear from UV-Vis spectral analysis that, increase in $\mathrm{CuO}$ concentration decreases the optical band gap from $4.978 \mathrm{eV}$ to $3.03 \mathrm{eV}$. The FTIR ( 3700 $650 \mathrm{~cm}^{-1}$ ) spectra of nanocomposite films are observed with changes by the addition of $\mathrm{CuO}$ nanomaterials.
\end{abstract}

\section{Keywords}

Nanocomposites, AC Conductivity, Dielectric Constant, XRD, UV-Analysis, FTIR

\section{Introduction}

Nanotechnology is rapidly evolving and expanding that has aroused growing media with public interest since from the past decade which has broad applications in many research areas, development and industrial activity. Development of new nanomaterials is a major theme of all of these programmes. It is established that inorganic addition to poly vinylalcohol (PVA) modifies its properties. This is of particular interest to science 
and technology and also it has many applications in modern engineering [1]. The nature of the interaction between metal ions and polymer molecules is usually approached by different techniques. AC conductivity of disordered polymers and insulators has been of significant interest recently because it provides information about the electronic structure [2]-[4]. We can obtain information about the dielectric constant, the mechanism of AC conduction and the activation energy from these measurements.

In semiconductor physics copper oxide is of good interest which forms two well-known stable oxides, i.e., cupric oxide $(\mathrm{CuO})$ and cuprous oxide $\left(\mathrm{Cu}_{2} \mathrm{O}\right)$. These two oxides have different physical properties and electrical properties. They also differ in colors and crystal structures. The possibility of low cost production methods and the good electrochemical properties make $\mathrm{CuO}$ to be one of the best materials for electrical, optical, sensing and so forth. $\mathrm{CuO}$ nanostructures have a wide range of applications in gassensors [5]-[7]. The objective of this paper is to study the effect of $\mathrm{CuO}$ concentration on dielectric behaviors of PVA with a view of understanding the conduction mechanism involved [8] [9].

\section{Experimental Part}

\subsection{Materials and Preparation of CuO Nanoparticles}

$\mathrm{CuO}$ nanoparticles are synthesized by aqueous precipitation method using copper (II) acetate $\left.\left[\mathrm{Cu}\left(\mathrm{CH}_{3} \mathrm{COO}\right)_{2} \cdot \mathrm{H}_{2} \mathrm{O}\right)\right]$ as parent and sodium hydroxide $(\mathrm{NaOH})$ as a decreasing agent. In short, $0.2 \mathrm{M}$ copper (II) acetate solution $(300 \mathrm{ml})$ and glacial acetic acid $\left(\mathrm{CH}_{3} \mathrm{COOH}\right)(1 \mathrm{ml})$ were added to a round-bottom flask and heated to boil under magnetic stirrer. In a flask, $15 \mathrm{ml}$ of $3 \mathrm{M} \mathrm{NaOH}$ solution was poured. The colour of the solution turned from blue to black immediately and a black suspension formed simultaneously. The reaction was carried out under stirring and boiling for $3 \mathrm{hr}$. The mixture was cooled to room temperature and centrifuged by which a wet $\mathrm{CuO}$ precipitate was obtained. The precipitate was filtered and washed with distilled water and absolute ethanol for several times. The resulting product was dried at $80^{\circ} \mathrm{C}$ for $8 \mathrm{hr}$ to obtain the dry powder of $\mathrm{CuO}$ nanoparticles. The yield of prepared $\mathrm{CuO}$ nanoparticles was $75 \%$.

\subsection{Synthesis of CuO Doped PVA Nanocomposites}

Pure PVA film and films doped with $\mathrm{CuO}$ nanoparticle at different concentrations were prepared by the solution casting method. First, the PVA stock solution was prepared and to this solution $1 \mathrm{wt}, 2 \mathrm{wt}, 3 \mathrm{wt}, 4 \mathrm{wt}$ and $5 \mathrm{wt}$ of $\mathrm{CuO}$ nanoparticles was mixed and stirred vigorously by using magnetic stirrer until transparent PVA-CuO multi component dispersion was obtained. Further, ultrasonification was done for complete dispersal of $\mathrm{CuO}$ in PVA solution. Appropriate mixtures of PVA and $\mathrm{CuO}$ solution were mixed and poured on to a level clean glass plate and left to dry at room temperature for about $48 \mathrm{hr}$. The dried films were peeled off from the glass plate and cut into suitable pieces for characterization.

\subsection{Instrumentation}

The AC conductivity studies and dielectric studies were carried out in the frequency 
range from $50 \mathrm{~Hz}$ to $5 \mathrm{MHz}$ by using HIOKI 3532-50 LCR HITESTER Version 2.4 and in the temperature range of 303 to $338 \mathrm{~K}$. The structural and morphological studies of $\mathrm{CuO}$ nanoparticles and $\mathrm{CuO}$ doped PVA nanocomposites were carried out using XRD and FTIR (3700 $-650 \mathrm{~cm}^{-1}$ ) analysis. The optical absorbance (A) of the samples was measured as function of wavelength $(\lambda)$ ranging from $(200-800 \mathrm{~nm})$ using computerized Spectrophotometer $2080 \mathrm{UV}$-Visible instrument.

\section{Results and Discussion}

\subsection{Electrical Studies}

The electrical properties of PVA-CuO nanocomposites films are influenced mainly by the synthesis technique, grain size, cation distribution etc. In the present studies, dielectric and $\mathrm{AC}$ conductivity studies have been undertaken on the as prepared $\mathrm{CuO}$ nanoparticles and $\mathrm{CuO}$ doped PVA nanocomposites. In particular, measurement of AC conductivity studies and dielectric constant $\left(\varepsilon^{\prime}\right)$ has been undertaken [10].

\subsubsection{AC Conductivity}

Material's conductivity depends on its overall characteristics such as its chemical composition, crystal structure, and purity. The continuous currents measurements provide only total conductivity. However, simultaneous data on conductivity and on the material's electrode interface effects can be partially offset by impedance measurements. Impedance measurements are a flexible tool for electrical characterization of materials. The Ohmic contacts were made using air drying silver paint on opposite faces of the prepared nanocomposite films and measurements are taken. The measured conductance $G(\omega)$ was used to calculate the AC conductivity of the sample.

$$
\sigma=\frac{G(\omega) d}{A}
$$

where, $G(\omega)$ is the measured conductance, $A$ is the area of the sample and $d$ is the thickness of the sample.

The variation of AC conductivity with frequency for pure PVA, $\mathrm{CuO}$ and $\mathrm{CuO}$ doped PVA nanocomposites is shown in Figure 1. There is a small increase in the electrical conductivity of $\mathrm{CuO}$ doped PVA at the low frequency region. But, at high frequencies especially in the $\mathrm{MHz}$ region, there is an abrupt increase in the conductivity. The high AC conductivity for $\mathrm{CuO}$ doped PVA could be attributed to small polaron hopping. The AC conductivity due to an alternating voltage across the samples was analyzed in order to understand their conductivity mechanism. For pure $\mathrm{CuO}$ nanoparticles the AC conductivity values are high compared to PVA-CuO nanocomposites. Figure 2 gives the plot of conductivity with temperature for 3 wt\% CuO doped PVA nanocomposites at $3 \mathrm{MHz}$ region, which shows that the conductivity increases with increase in temperature.

\subsubsection{Dielectric Studies}

The dielectric constant is crucial parameter required in the design of electronic and 


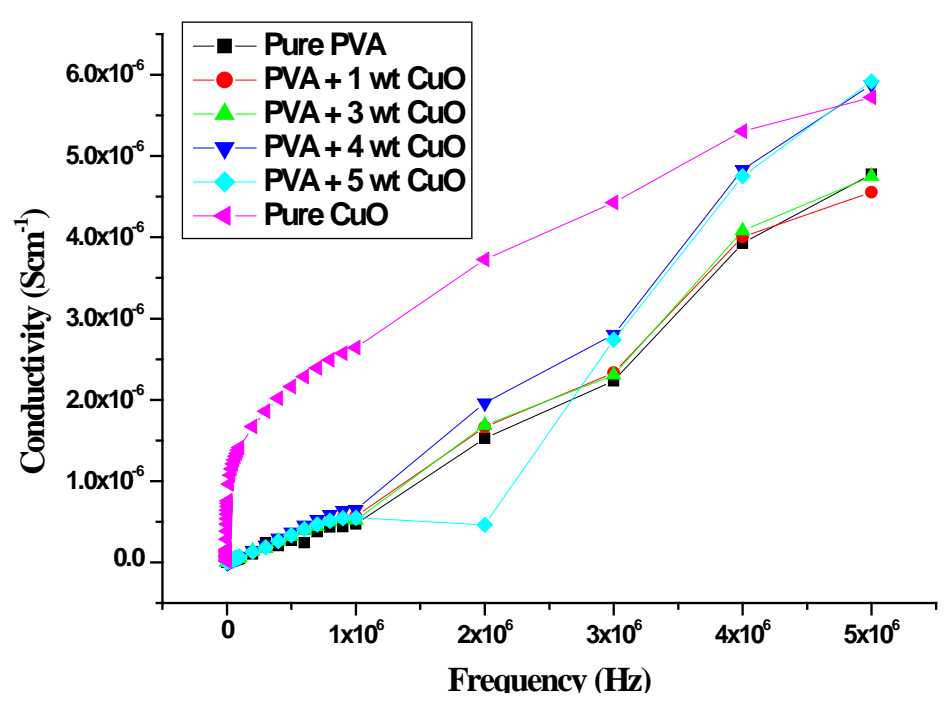

Figure 1. Variation of conductivity with frequency for PVA, $\mathrm{CuO}$ and PVA-CuO nanocomposites.

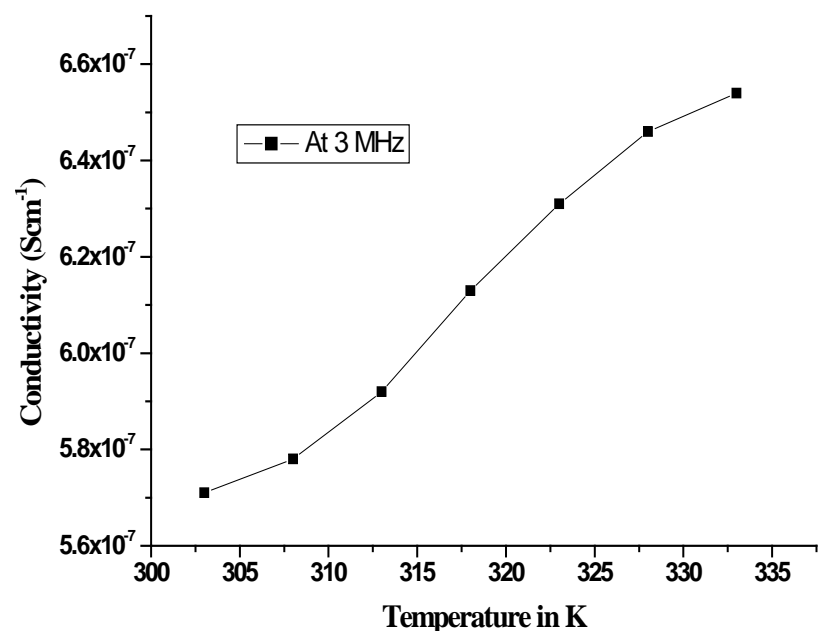

Figure 2. Variation of AC conductivity with temperature for $3 \mathrm{wt} \% \mathrm{CuO}$ doped PVA nanocomposites at $3 \mathrm{MHz}$.

electrical devices and furthermore, as a function of temperature or frequency, they reveal much information on the chemical or physical state of the polymer [11]. The dielectric constant is calculated from the measured values of $\mathrm{Cp}$ for various frequencies at room temperature. The real part of the dielectric constant was calculated using the relation,

$$
\varepsilon^{\prime}=\frac{C d}{\varepsilon_{0} A}
$$

where, $C$ is the capacitance and $\varepsilon_{o}$ is the permittivity of the free space $\left(8.854 \times 10^{-12}\right.$ $\mathrm{F} / \mathrm{m})$.

The decrease in dielectric constant with frequency can be attributed to the decrease in polarization with frequency. At very low frequencies $(\omega \ll 1 / \tau, \tau$ is the relaxation 
time), dipoles follow the field and $\varepsilon^{\prime}=\varepsilon^{s}$ (value of the dielectric constant at quasi-static field). As the frequency increases (with $\omega<1 / \tau$ ), dipoles begin to lag behind the field and $\varepsilon^{\prime}$ slightly decreases. When the frequency reaches the characteristic frequency $(\omega=1 / \tau)$ dielectric constant drops. Similar trend of variation of dielectric constant with frequency was observed for $\mathrm{CuO}$ doped PVA nanocomposites (Figure 3) [12]. For pure $\mathrm{CuO}$ nanoparticles the dielectric constant values are low compared to PVA-CuO nanocomposites. After $1 \times 10^{6} \mathrm{~Hz}$ region we have taken readings for 2, 3, 4 and $5 \times 10^{6} \mathrm{~Hz}$ to study the variation at high frequency region.

\subsection{Optical Studies}

\subsubsection{XRD Studies}

$\mathrm{X}$-ray diffraction (XRD) technique is used for materials characterization as it provides important information about the internal structure of matter such as crystallite size, nature of the solid sample (amorphous or crystalline), atomic spacing and crystal structure. Figure 4 shows the XRD spectra of $\mathrm{CuO}$ nanoparticles.

The sharp peaks and the crystallite size of $\mathrm{CuO}$ nanopowder is calculated by using Debye-Scherrer formula is as shown in the Table 1 [13],

$$
D=\frac{k \lambda}{\cos \theta \beta}
$$

where, $k$ is the Scherrer constant $(k=0.9)$, which is related to the crystallite shape, $\lambda$ and $\theta$ are the radiation wavelength and Bragg's angle, respectively; and $\beta$ is the full width at half maximum of the diffraction peak.

The peak widths in XRD pattern of PVA-CuO nanocomposites are broadened by incremental addition of $\mathrm{CuO}$ nanomaterials is as shown in Figure 5 which implies an increase in the amorphous character of PVA-CuO nanocomposites. This amorphous nature results in greater ionic diffusivity and high ionic conductivity. The crystalline

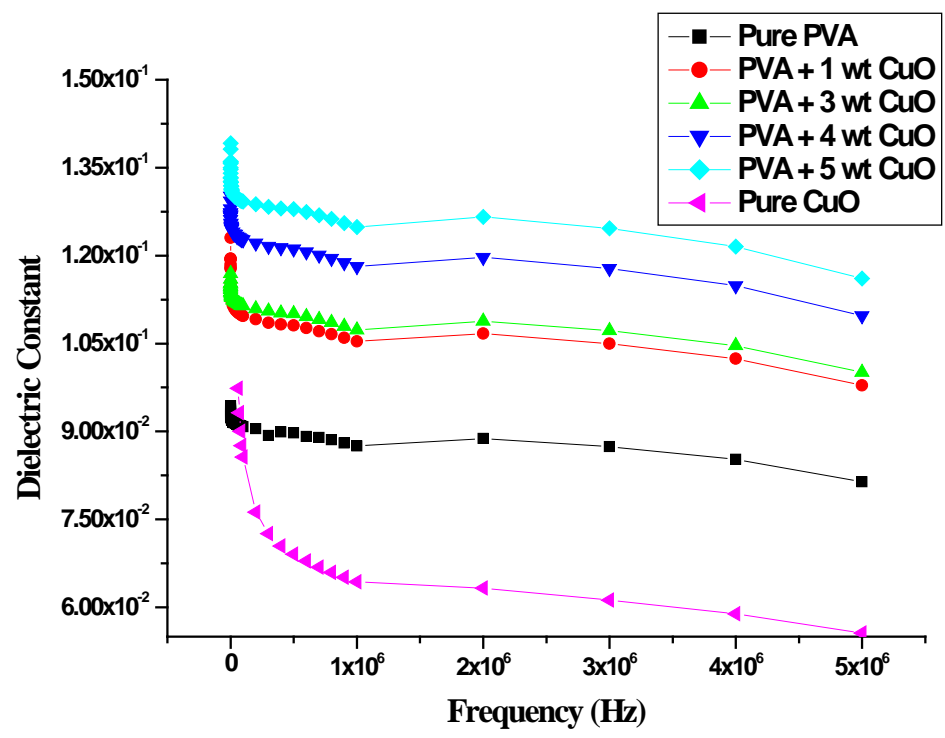

Figure 3. Plot of dielectric constant V/s frequency of PVA, $\mathrm{CuO}$ and PVA-CuO nanocomposites. 


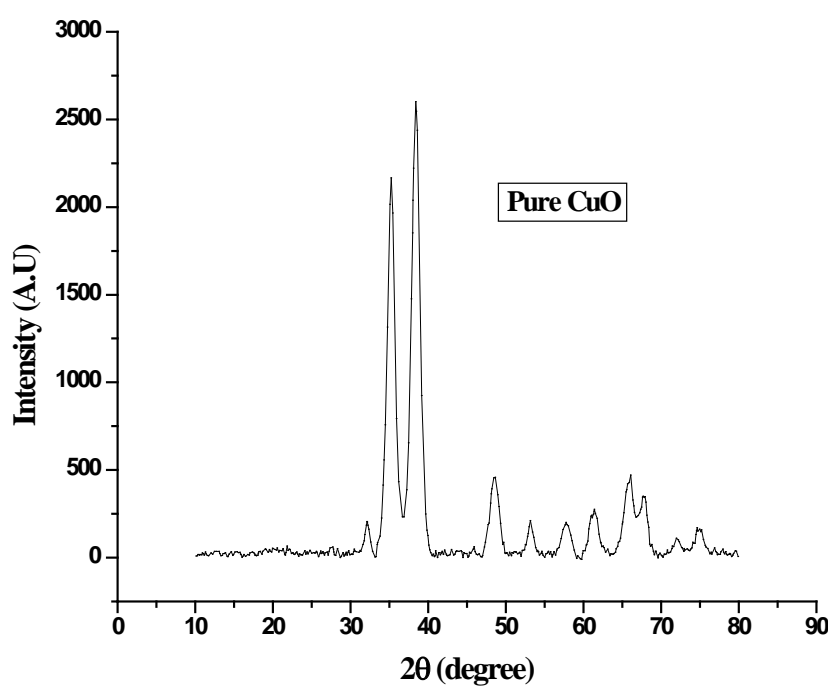

Figure 4. XRD spectra of $\mathrm{CuO}$ nanoparticles.

Table 1. XRD peak and crystalline size of $\mathrm{CuO}$ nanopowder.

\begin{tabular}{cccccc}
\hline Peak & $2 \theta$ (degree) & $\mathrm{d}(\AA)$ & $\beta$ in degree & $\beta$ in radians & Ds nm \\
\hline 1 & 35.25 & 2.561 & 0.99 & 0.0174 & 8.36 \\
2 & 38.37 & 2.343 & 1.06 & 0.0185 & 7.91 \\
\hline
\end{tabular}

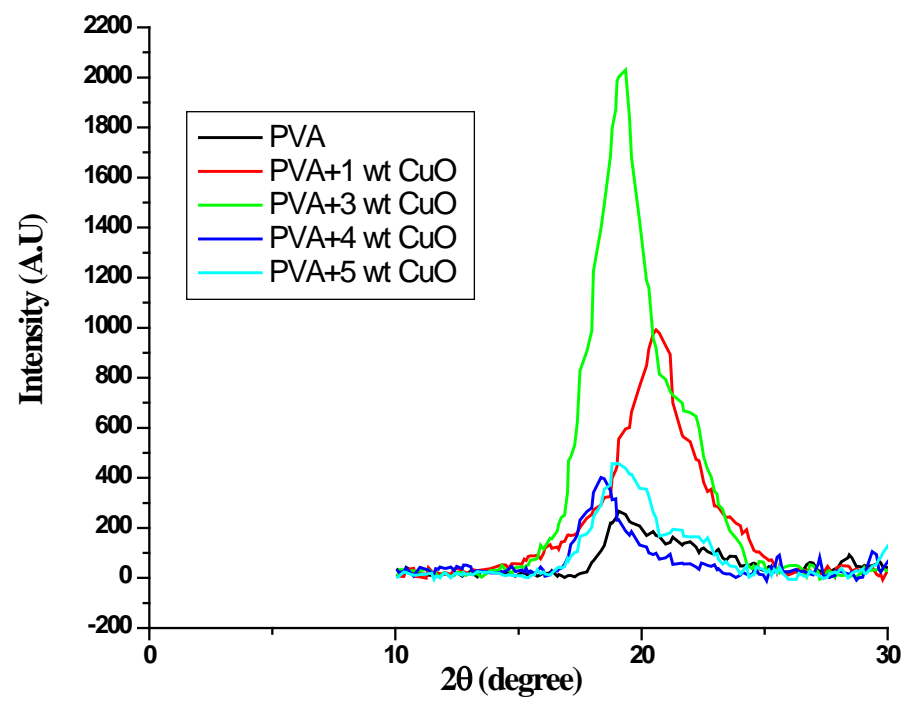

Figure 5. XRD spectra of PVA/PVA-CuO nanocomposites.

nature of prepared $\mathrm{CuO}$ nanomaterials is observed by the various sharp crystalline peaks in the XRD pattern. The absence of shifting at the locations of the peak in the $\mathrm{XRD}$ pattern of PVA-CuO and the absence of the peaks characterizing $\mathrm{CuO}$ nanomaterials in all studied PVA-CuO nanocomposites indicates that incorporated $\mathrm{CuO}$ nanomaterials lose their structural registry and disperse into the polymer matrix, producing exfoliated nanocomposite, i.e., nano-fillers dispersed into the polymer matrix [14]. 


\subsubsection{UV-Vis Studies}

The optical absorption [15] is an important tool to obtain optical energy band gap of crystalline and amorphous materials. The fundamental absorption corresponds to the electron excitation from the valence band to the conduction band can be used to determine the nature and value of the optical band gap. The absorption spectrum reveals that the increase in concentration of $\mathrm{CuO}$ produces shift in the absorption peak and results in low band gap. The pure PVA film has remarkable absorption in the visible light region with a wavelength above $400 \mathrm{~nm}$ whereas the $\mathrm{CuO}$ dispersed nanocomposites films extend the absorption spectrum into the visible region. The absorption peak at UV region, were used to study the shifting in the optical energy band gap for PVA and PVA-CuO films at $25^{\circ} \mathrm{C}$. The optical energy band gap $E g$ is calculated from the relation:

$$
(\alpha h v)=B(h v-E g)^{n}
$$

where $h v$ is the photon energy, $B$ is the constant and $n$ is the power factor and that takes $\frac{1}{2}, 2, \frac{3}{2}$ and 3 allowed direct, allowed indirect, forbidden direct and forbidden indirect transitions respectively.

Table 2 gives an optical band gap value which shows that, $\mathrm{CuO}$ addition decreases the optical band gap from $4.97 \mathrm{eV}$ to $3.03 \mathrm{eV}$ with increasing concentration shown in Figure 6 and Figure 7 which is similar to that observed for PVA-CuI composite films

Table 2. Values of optical band gap for PVA-CuO nanocomposites.

\begin{tabular}{cc} 
Samples & $\begin{array}{c}\text { Optical band gap } \\
(E g) \text { in eV }\end{array}$ \\
Pure PVA & 4.97 \\
PVA + 1 wt CuO & 4.88 \\
PVA + 2 wt CuO & 4.25 \\
PVA + 3 wt CuO & 4.00 \\
PVA + 4 wt CuO & 3.90 \\
PVA + 5 wt CuO & 3.03 \\
\hline
\end{tabular}

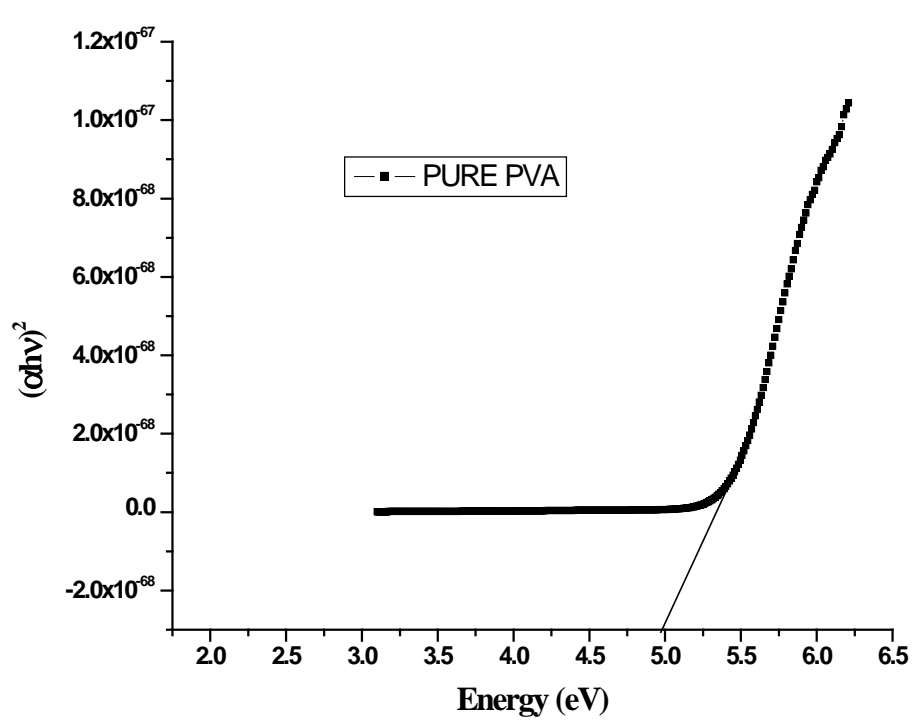

Figure 6. The plot of $(a h v)^{2}$ versus energy (hv) of pure PVA. 
[16]. This may be attributed to salt complexation with polymer matrix besides the expected nanoparticles aggregation. The absorption increases and optical band gap decreases due to charge transfer transitions [17] [18].

Figure 8 shows the measurements of optical and electrical conductivity which indicates decreases of band gap and increases of $\mathrm{AC}$ conductivity with $\mathrm{CuO}$ dispersion. The prepared PVA-CuO nanocomposites in the present study expected to be more useful in photonic and electronic device applications.

\subsection{FTIR Studies}

FTIR can be used to analyze a wide range of materials which are in bulk or thin films, liquids, solids, pastes, powders, fibers and other forms. FTIR analysis can give not only qualitative (identification) analysis of materials, but with suitable standards, can be

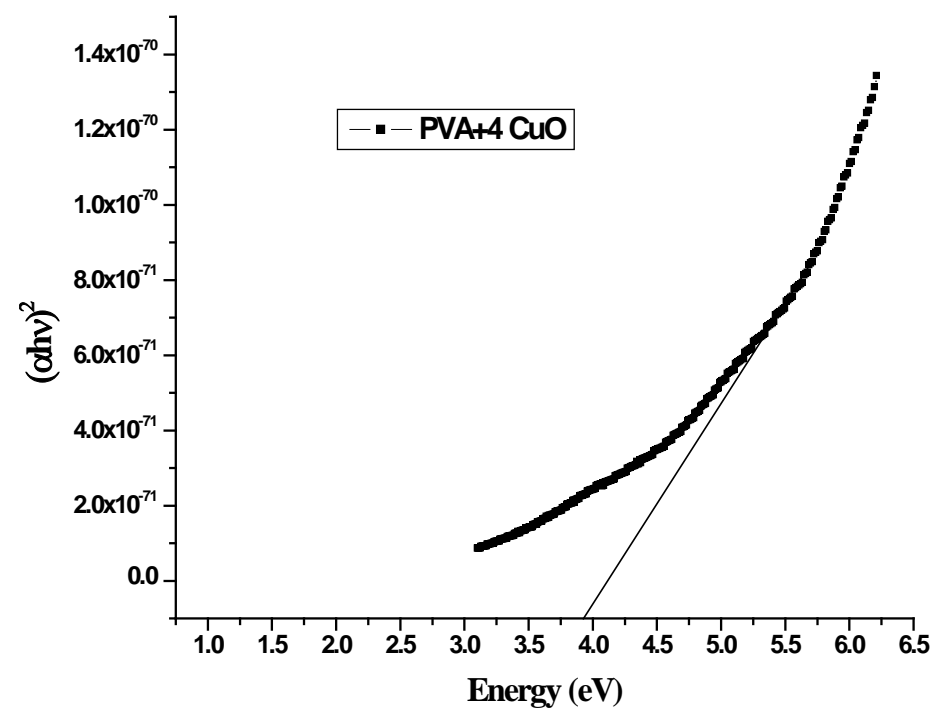

Figure 7. The plot of $(\alpha h v)^{2}$ versus energy (hv) of 4 wt CuO doped PVA nanocomposites.

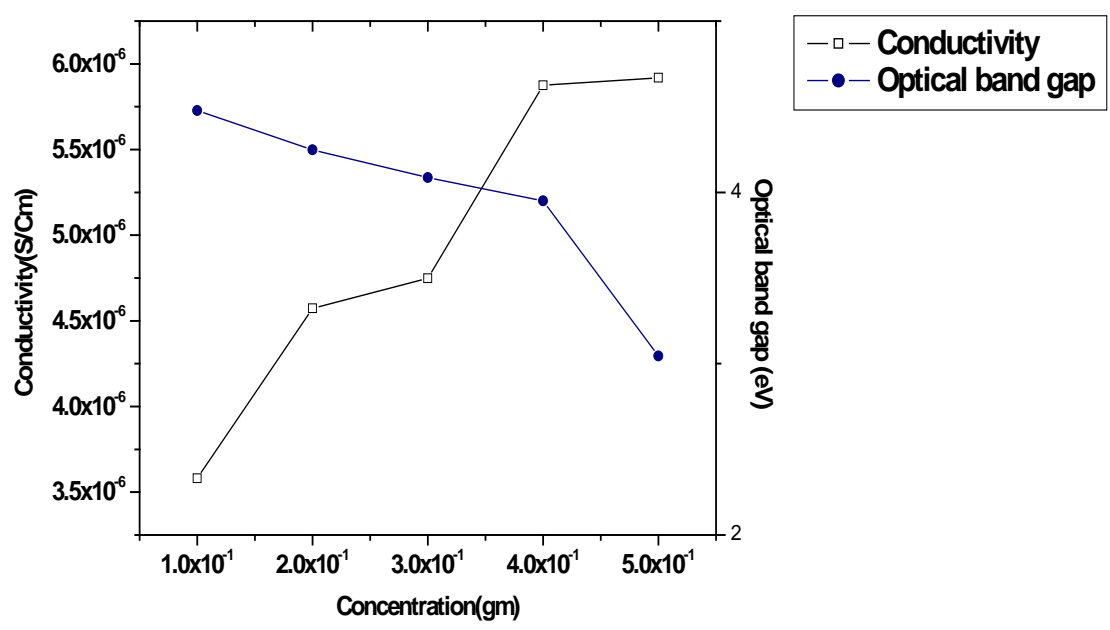

Figure 8. The variation of conductivity and optical band gap as a function of dopant concentration. 
used for quantitative (amount) analysis. FTIR spectra were recorded for all the prepared PVA-CuO nanocomposites films at room temperature in frequency region 600 $4000 \mathrm{~cm}^{-1}$ and the spectra is shown in Figure 9.

There are two absorbance bands which appear at around 650 and $3600-3100 \mathrm{~cm}^{-1}$ and sharp absorption band at $672.85 \mathrm{~cm}^{-1}$ is associated with $\mathrm{Cu}-\mathrm{O}$ stretching mode. A broad band in the range of $3500-3000 \mathrm{~cm}^{-1}$ is due to the stretching in water molecules associated with $\mathrm{CuO}$. FTIR result suggests the presence of $\mathrm{Cu}-\mathrm{O}$ bonds and some constitutional water is incorporated in the copper oxide structure. Thus, the formation of copper oxide is confirmed from the FTIR study.

In FTIR spectra of PVA-CuO nanocomposites films, characteristic bands of PVA are observed with two significant changes by the addition of $\mathrm{CuO}$ nanomaterials. i.e., slight shifting in vibrational bands and slight changes in intensities of absorption bands at 1753.87 and $1656.47 \mathrm{~cm}^{-1}$. First, the vibrational bands of PVA are slightly shifted by

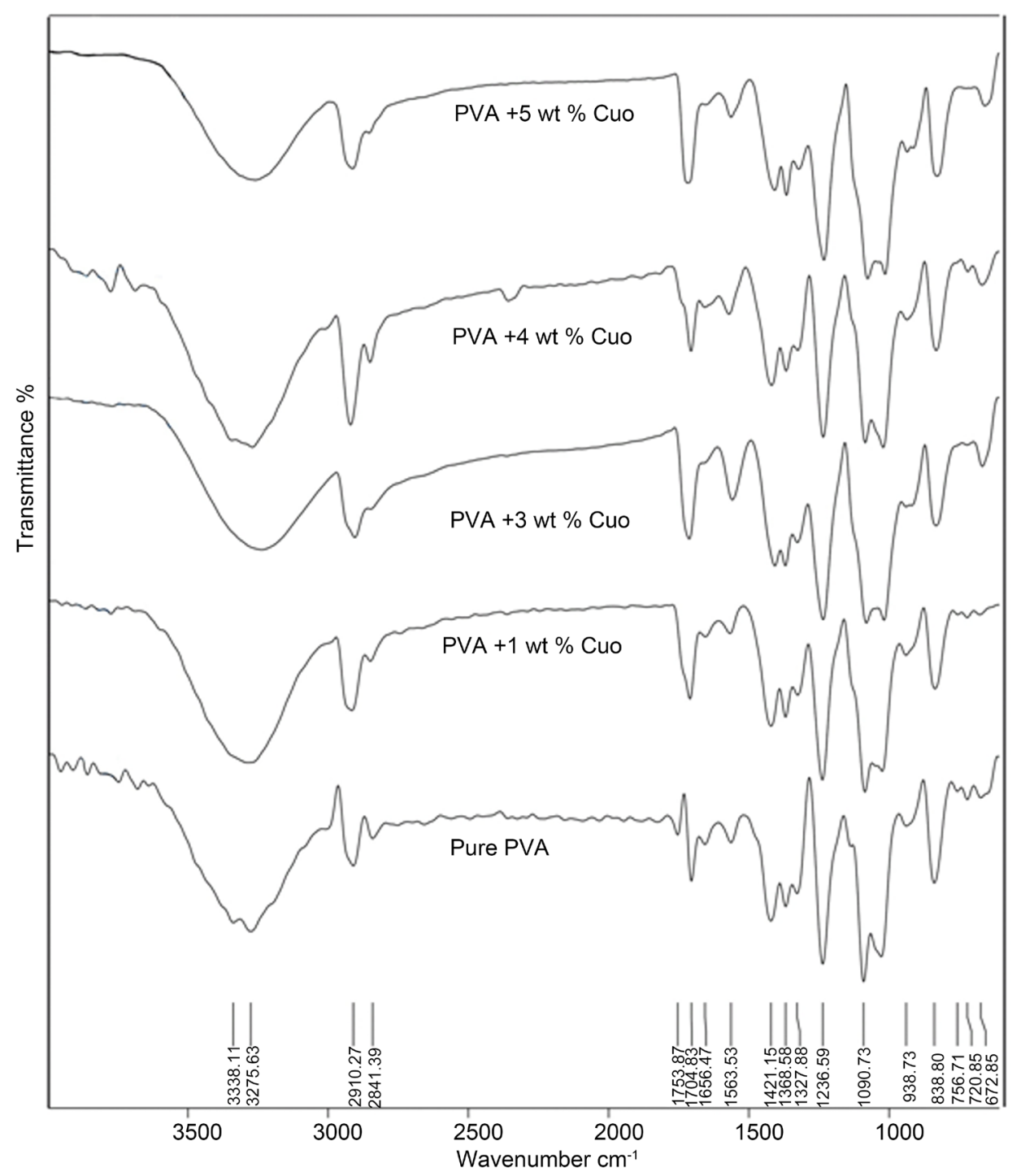

Figure 9. FTIR spectra of PVA/PVA-CuO nanocomposites. 
the addition of $\mathrm{CuO}$ nanomaterials. However, the values are within spectra range of pristine PVA. Secondly, structural changes in the PVA molecules due to the addition of $\mathrm{CuO}$ nanomaterials are observed by two remarkable changes in the intensity of absorption peaks. These are the increasing the intensity of $\mathrm{C}=\mathrm{O}$ and $\mathrm{C}=\mathrm{C}$ bands at 1753.87 $\mathrm{cm}^{-1}$ and $1656.47 \mathrm{~cm}^{-1}$ respectively. Such changes are possible only with a new generation of these groups.

\section{Conclusion}

We have prepared pellets of $\mathrm{CuO}$ nanoparticles and films of $\mathrm{CuO}$ doped PVA nanocomposites. Results of AC electrical conductivity measurements observed in this study indicates an increase of electronic conductivity and decrease of the dielectric constant with the increase in concentration of $\mathrm{CuO}(1 \mathrm{wt}, 2 \mathrm{wt}, 3 \mathrm{wt}, 4 \mathrm{wt}$ and $5 \mathrm{wt})$ dispersion in the PVA polymer matrix. The peak widths in XRD pattern of PVA-CuO nanocomposites are broadened by incremental addition of $\mathrm{CuO}$ nanomaterials indicating an increase in the amorphous character of PVA-CuO nanocomposites. This amorphous nature results in greater ionic diffusivity and high ionic conductivity. From FTIR analysis, the vibrational bands of PVA are slightly shifted by the addition of $\mathrm{CuO}$ nanomaterials which indicates that the considerable interaction exists between PVA matrix and $\mathrm{CuO}$ nanomaterials. By UV-Visible studies, the absorption spectrum reveals that the increase in concentration of $\mathrm{CuO}$ produces shift in the absorption peak and results in low band gap. Thus, the present study indicates that $\mathrm{CuO}$ dispersion tunes the optical absorption property of PVA significantly and consequently makes the PVA film more useful. The prepared PVA-CuO nanocomposites in the present study are expected to be more useful in photonic and electronic device applications.

\section{Acknowledgements}

One of the author's (A. Manjunath) thanks University Grants Commission (UGC), New Delhi (Minor Research Project) for financial support.

\section{References}

[1] Sperling, L.H. (1992) Introduction to Physical Polymer Science. Wiley-Inter Science, New York.

[2] Veeravazhuthi, V., Narayan Dass, Sa.K. and Mangolaraj, D. (1998) Dielectric Behaviour of Pure and nickel-Doped Cellulose Acetate Films. Polymer International, 45, 383-385. http://dx.doi.org/10.1002/(SICI)1097-0126(199804)45:4<383::AID-PI949>3.0.CO;2-T

[3] Khare, P.K., Upadhayay, J.K., Verma, A. and Paliwal, S. (1998) Electrical Conduction Behaviour of Diphenylthiocarbazone Doped Cellulose Acetate. Polymer International, 47, 145151. http://dx.doi.org/10.1002/(SICI)1097-0126(1998100)47:2<145::AID-PI37>3.0.CO;2-M

[4] Diaz-Calleja, R., Friederichs, S., Jaimes, C., Sanchis, M.J., Belana, J., Canadas, J.C., Diego, J.A. and Mudarra, M. (1998) Comparative Study of Mechanical and Electrical Relaxations in Poly(etherimide) Part 2. Polymer International, 46, 20-28. http://dx.doi.org/10.1002/(SICI)1097-0126(199805)46:1<20::AID-PI944>3.0.CO;2-U

[5] Anderson, P.W. (1958) Absence of Diffusion in Certain Random Lattices. Physical Review, 109, 1492-1505. http://dx.doi.org/10.1103/PhysRev.109.1492 
[6] Anderson, P.W., Halperin, B.I. and Varma, C.M. (1972) Anomalous Low-Temperature Thermal Properties of Glasses and Spin Glasses. Philosophical Magazine, 25, 1-9. http://dx.doi.org/10.1080/14786437208229210

[7] Mott, N.F. and Davis, E.A. (1970) Electronic Processes in Non-Crystalline Solids. Clarendon, Oxford.

[8] Mishra, V., Thomas, S.C. and Nath, R. (1998) DC Conduction Studies in Amylase. Polymer International, 47, 331-334. http://dx.doi.org/10.1002/(SICI)1097-0126(199811)47:3<331::AID-PI54>3.0.CO;2-S

[9] Khare, P.K., Keller, J.M., Gaur, M.S., Singh, R. and Datt, S.C. (1994) Electrical Conductivity in Iodine-Doped Ethyl Cellulose. Polymer International, 35, 337-343. http://dx.doi.org/10.1002/pi.1994.210350406

[10] Indulal and Raveendran (2010) Indian Journal of Pure and Applied Physics, 48, 121-126.

[11] Abd El-Kader, F.H., Osman, W.H., Mahmoud, K.H. and Basha, M.A.F. (2008) Dielectric Investigations and AC Conductivity of Polyvinyl Alcohol Films Doped with Europium and Terbium Chloride. Physica B: Condensed Matter, 403, 3473-3484. http://dx.doi.org/10.1016/j.physb.2008.05.009

[12] Kulanthaisami, S., Mangalaraj, D. and Narayandass, Sa.K. (1995) Conduction Studies on Polyvinyl Alcohol Films. European Polymer Journal, 3, 969-975.

[13] Suramwar, N.V., Thakare, S.R. and Khaty, N.T. (2012) Synthesis and Catalytic Properties of Nano CuO Prepared by Soft Chemical Method. International Journal of Nano Dimension, 3, 75-80.

[14] Dhineshbabu, N.R., Rajendran, V., Nithyavathy, N. and Vetumperumal, R. (2016) Study of Structural and Optical Properties of Cupric Oxide $(\mathrm{CuO})$ Nanoparticles. Applied Nanoscience, 6, 933. http://dx.doi.org/10.1007/s13204-015-0499-2

[15] Essick, J.M. and Mather, R.T. (1993) Characterization of a Bulk Semiconductors Bandgap via Near Absorption Edge Optical Transmission Experiment. American Journal of Physics, 61, 646-649. http://dx.doi.org/10.1119/1.17173

[16] El-Mansy, M.K., Sheha, E.M., Patel, K.R. and Sharma, G.D. (2012) Characterization of PVA/CuI Polymer Composites as Electron Donor for Photovoltaic Application. Optik, 124, 1624-1631. http://dx.doi.org/10.1016/j.ijleo.2012.05.009

[17] Asha Radhakrishnan, A. and Baskaran Beena, B. (2014) Structural and Optical Absorption Analysis of $\mathrm{CuO}$ Nanoparticles. Indian Journal of Advances in Chemical Science, 2, 158161. http://www.ijacskros.com/artcles/IJACS-M64.pdf

[18] Gomathi, S.S., Vanathi Nachiyar, G.K. and Nandhini, P. (2016) Effect of Surfactant on the Structural and Optical Properties of $\mathrm{CuO}$ Nanoparticles. International Journal of Scientific Reseach, 5, 339-341. http://worldwidejournals.in/ojs/index.php/ijsr/article/view/1190/1191 
Submit or recommend next manuscript to SCIRP and we will provide best service for you:

Accepting pre-submission inquiries through Email, Facebook, LinkedIn, Twitter, etc.

A wide selection of journals (inclusive of 9 subjects, more than 200 journals)

Providing 24-hour high-quality service

User-friendly online submission system

Fair and swift peer-review system

Efficient typesetting and proofreading procedure

Display of the result of downloads and visits, as well as the number of cited articles

Maximum dissemination of your research work

Submit your manuscript at: http://papersubmission.scirp.org/

Or contact ampc@scirp.org 